\title{
Hierarchical Neural Network Based Compression of ECG Signals
}

\author{
Bekir Karlik \\ Department of Computer Engineering, University of Bahrain, P.O. Box: 32038, \\ Kingdom of Bahrain \\ bkarlik@itc.uob.bh
}

\begin{abstract}
Electrocardiogram (ECG) data compression algorithm is needed that will reduce the amount of data to be transmitted, stored and analyzed, but without losing the clinical information content. An example of application of hierarchical neural network structure is described for compression of ECG signals. Then results of this lossy compression method were compared with two efficient compression methods that are fractal based and wavelet based compressions.
\end{abstract}

\section{Introduction}

ECG signals are electrical measurements of heartbeat rhythms. The continuing proliferation of computerized electrocardiogram (ECG) processing systems along with the increased feature performance requirements and demand for lower cost medical care have mandated reliable, accurate, and more efficient ECG data compression techniques. The practical importance of ECG data compression has become evident in many aspects of computerized electrocardiography including: a)increased storage capacity of ECG's as databases for subsequent comparison or evaluation, b) feasibility of transmitting real-time ECG's over the public phone network, c) implementation of cost effective real-time rhythm algorithms, d)economical rapid transmission of off-line ECG's over public phone lines to a remote interpretation center, and e) improved functionality of ambulatory ECG monitors and recorders [1].

Recently some of the frequency domain techniques offer good compression ratios, generally at the expense of more computations [1, 2, 3]. G.E. Oien et al. proposed a fast compression method for ECG signal [4]. But this one also still has more computation, and has not high compression ratio. I. Provaznik and J. Kozumplik's method has good compression ratio and reconstructed [5]. But it has more computation than Oien's work and this work.

In this work, utilizing hierarchical neural networks ECG data compression is done by software. In teaching mode, ECG signals are applied both input and output of ANN structure by using the principle of ANN work. So, obtained weight parameters provided a base for next ECG samples. Also two efficient methods of data compression, wavelet and fractal based compression (proposed in reference [4-5]) were compared with proposed method. This direct data compression technique for ECG signals has shown a more efficient performance than the transformation 
techniques in regard particularly to processing speed and generally to compression ratio.

\section{Background of ECG Signal Compression}

The main goal of any compression technique is to achieve maximum data volume reduction while preserving the significant signal morphology features upon reconstruction. Conceptually, data compression is the process of detecting and eliminating redundancies in a given data set. Redundancy in a digital signal exists whenever adjacent signal samples are statistically dependent and/or the quantized signal amplitudes do not occur with equal probability. However, the first step towards ECG data compression is the selection of minimum sampling rate and word length consequently further compression of the ECG signal can be achieved by exploiting the known statistical properties of the signal.

While it is done any comparison among ECG data compression techniques, the following properties are determined:

1) Signal sampling frequency $\left(\mathrm{f}_{0}\right)$ :

In analog/digital converter utilized to convert ECG signals to digital the sampling frequency can be different frequencies in accordance with purpose but generally has been selected at $500 \mathrm{~Hz}$.

2) Bit numbers in digital samples (p,"precision"):

Bit numbers which show resolution of stored ECG data can be 8 or 12 bits.

3) Compression ratio (CR):

This is one of the important parameters in data compression algorithms and the large value of this ratio shows success of any algorithm.

$$
\mathrm{CR}=\frac{\text { The number of samples before compression }}{\text { The number of samples after compression }}
$$

4) Performance index (PRD, "Percent Root Mean Square Difference"):

PRD is other one of the important parameters of any algorithms and the small value of PRD shows success of algorithm.

$$
P R D=\sqrt{\frac{\sum_{i=1}^{n}\left[X_{\text {org }}(i)-X_{\text {rec }}(i)\right]^{2}}{\sum_{i=1}^{n}\left[X_{\text {org }}(i)\right]^{2}}} \cdot 100
$$

where $\mathrm{X}_{\mathrm{org}}$ and $\mathrm{X}_{\mathrm{rec}}$ are samples of the original and reconstructed data sequences.

5) The higher speed of any algorithm is, the quicker the process can be done and realtime studies can be done.

6) Most of the databases utilized in evaluating ECG compression algorithms are non standard. However the algorithm results can be different in accordance with databases. 


\subsection{Neural Networks Based ECG Signal Compression}

Artificial Neural Networks (ANN) can use for 1D or 2D signal compression and reconstruction [6-7]. The basic back-propagation network can be further extended to construct a hierarchical neural network by adding two more hidden layers into the existing network, in which the three hidden layers are termed as combiner layer, compressor layer and decombiner layer. The structure can be shown in Figure 1. The idea is to exploit correlation between pixels by inner hidden layer and to exploit correlation between blocks of pixels by outer hidden layers. From input layer to combiner layer and decombiner layer to output layer, local connections are designed, which has the same effect as $\mathrm{M}$ fully connected neural sub-networks.

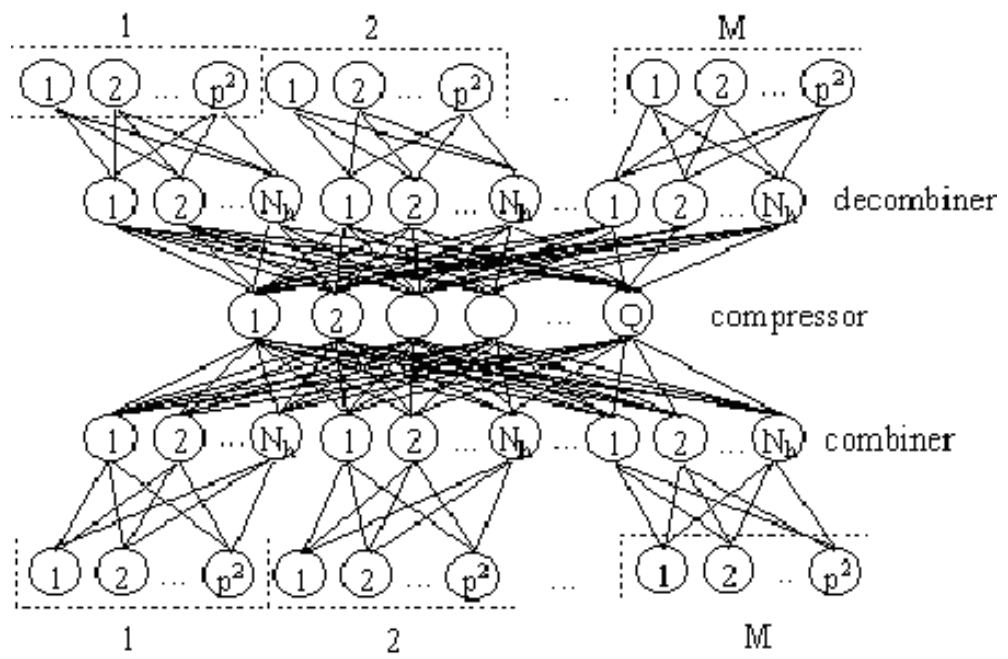

Training of such a neural network can be conducted in terms of: (i) Outer Loop Neural Network (OLNN) Training; (ii) Inner Loop Neural Network (ILNN) Training; and (iii) Coupling weight allocation for the Overall Neural Network

Fig. 1. The structure hierarchical neural network

\subsection{Fractal Based ECG Signal Compression}

Fractal compression exploit block wise self-similarity in the signal, which is split into non-overlapping range blocks to be coded. Each range is approximated by the decimated and nonlinearly transformed domain which is the most similar to the range, out of all the domain candidates in the signal [8].

Formally, a signal $\mathrm{x}$ (M samples long) is thus encoded as the collage Tx of an affine contractive map $\mathrm{T}: \mathrm{X} \rightarrow \mathrm{X}$ on $\mathrm{X}=\mathrm{R}^{\mathrm{M}}$. T consists of a linear part $\mathrm{L}: \mathrm{X} \rightarrow \mathrm{X}$, and an offset $\mathrm{t}$ : 


$$
T x=L x+t=\left(\sum_{n=1}^{N_{r}} \alpha_{2}^{(n)} P_{n} O_{n} D_{n} F_{d(n)}\right) x+\sum_{n=1}^{N_{r}} \alpha_{1}^{(n)} P_{n} b_{1}^{(n)}
$$

where $\mathrm{N}_{\mathrm{r}}$ is the number of ranges. $\mathrm{F}_{\mathrm{d}(\mathrm{n})}: \mathrm{X} \rightarrow \mathrm{R}^{\mathrm{Dn}}$ fetches the best domain for range $\mathrm{n}$. $D_{n:} R^{D n} \rightarrow R^{B n}$ decimates this domain to range length $B_{n}$ by simple averaging $\left(D_{n} / B_{n}=2\right) . O_{n}: R^{B n} \rightarrow R^{B n}$ orthogonalizes the decimated domain with respect to the offset basis vector $\mathrm{b}_{1}{ }^{(\mathrm{n})}$, a constant (DC) block. $\mathrm{P}_{\mathrm{n}}: \mathrm{R}^{\mathrm{Bn}} \rightarrow \mathrm{X}$ places the sum of offset and transformed domain in range position $\mathrm{n}$. Here, $\alpha_{1}^{(n)}, \alpha_{2}^{(n)}$ are scaling for the offset and transformed domain respectively. The code for each range now consists of $\alpha_{1}^{(n)}, \alpha_{2}^{(n)}$, and d(n). The decoded signal is the attractor of T;

$$
x_{T}=\sum_{k=0}^{K-1} L^{k} t
$$

where $\mathrm{K}$ is big enough to ensure convergence. The decoded attractor will be almost identical to encoder collage approximation (by the Collage Theorem [8]).

\subsection{Wavelet Based ECG Signal Compression}

The wavelet coefficients are computed in float point operation arithmetic. To store the data efficiently and increase compression ratio, the coefficients are represented as integers with predefined word length. A quantization step corresponds to range of coefficients values for fixed word length and implies resulting error.

The dyadic discrete time wavelet transform (DTWT) of a finite sequence $\{\mathrm{x}(\mathrm{i}) \mid \mathrm{i}=0,1, \ldots, \mathrm{N}-1\}$, where $\mathrm{N}=2^{\mathrm{M}}$, can be evaluated as cyclic convolution;

$$
y(m, n)=\operatorname{DFT}_{N, 2^{m}}^{-1}\left[X(k) H_{m}(k)\right]
$$

where $\quad \mathrm{m}=1,2, \ldots, \mathrm{M} ; \quad \mathrm{n}=0,1, \ldots, \mathrm{N} / 2^{\mathrm{m}}-1 ; \quad \mathrm{k}=0,1, \ldots, \mathrm{N}-1 ; \quad \mathrm{X}(\mathrm{k})=\mathrm{DFT}[\mathrm{X}(\mathrm{i})] \quad$ and $\mathrm{H}_{\mathrm{m}}(\mathrm{k})=\left(2^{\mathrm{m}}\right)^{1 / 2} \mathrm{G} *\left(2^{\mathrm{m}} \mathrm{k}\right)$ is a sampled frequency characteristic of $\mathrm{m}$-th filter that corresponds to a proper expanded mother wavelet. $\mathrm{N}, 2^{\mathrm{m}}$ index means $\mathrm{N}$-point inverse discrete Fourier transform where every $2^{\mathrm{m}}$-th sample of output signal $\mathrm{y}(\mathrm{m}, \mathrm{n})$ is chosen. Use of orthogonal set of wavelets is necessary to transform the signal without any error. In this study, Meyer's wavelets that are originally defined in frequency domain as described in [9], is used.

\section{Results}

In this work, using hierarchical neural network compression and reconstructed of the some ECG's are done. These ECG's are from MIT/BIH database tapes 100, 107 and 109. Tapes 100, 107 and 109 are respectively normal, paced beat and left bundle 
branch block ECG's. Original data sampled at $360 \mathrm{~Hz}$ and this sampling rate is decreased to $200 \mathrm{~Hz}$ in this work.

Compression processing which is run on computer in Borland $\mathrm{C}$ is provided, as above mentioned, with 200 input nodes, 10 and 5 hidden nodes and 200 output nodes. At the same time, these values give compression ratio, i.e. 20:1 and 40:1. Therefore the utilized ANN structures are as 200:10:200 and 200:5:200. Generally, 20 cycles from ECG's are used in training process. As above mentioned, learning coefficient $(\varepsilon)$ and momentum $(\alpha)$ must be selected to minimize the error in training process.

Reconstructed processing for compressed ECG is provided with 10 input nodes (because of 10 hidden nodes in compression) or 5 input nodes (because of 5 hidden nodes in compression) and 200 output nodes. For normal ECG, original and reconstructed ECG is shown in Figure 2. An advantage of this technique is to compress large numbers of data, i.e. 10 cycles (samples) from ECG's are used for training and 100 cycles (samples) can be compressed.

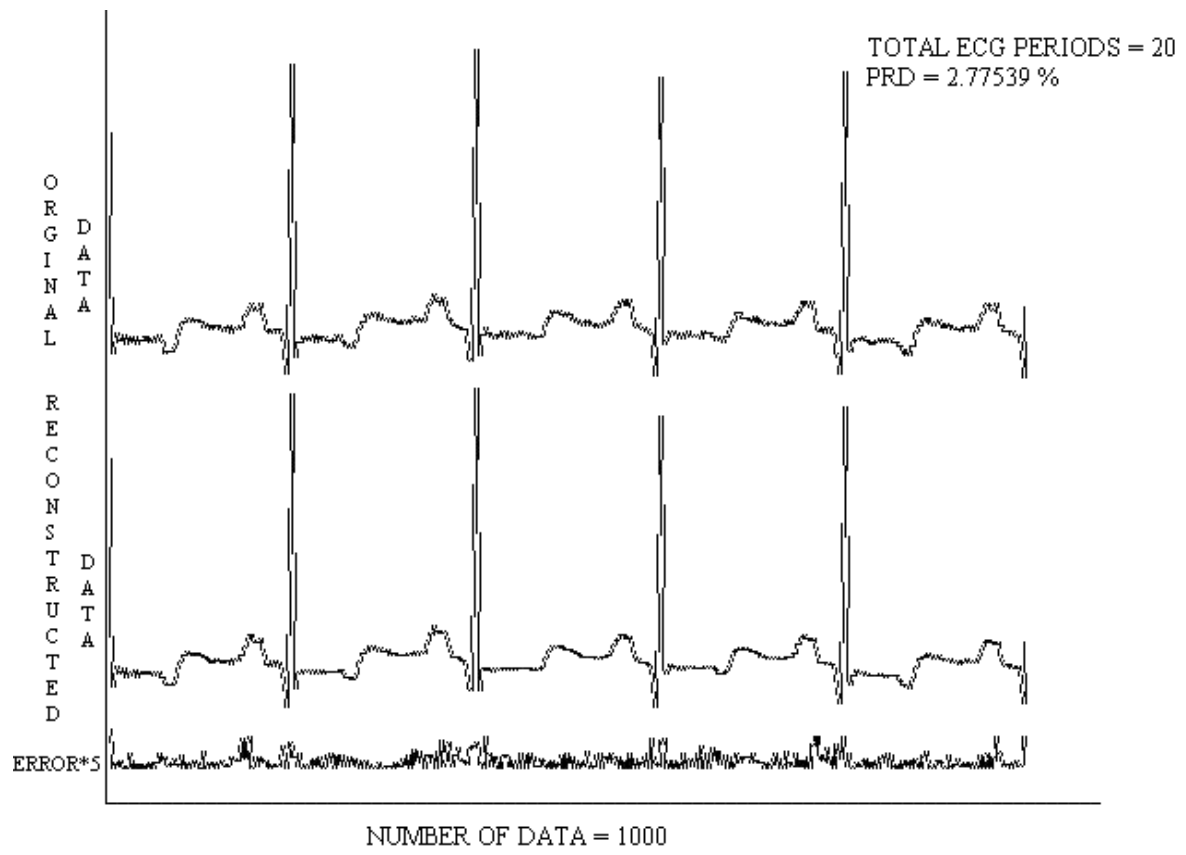

Fig. 2. Graphics of the original and reconstructed ECG from MIT/BIH tapes 100 for 10 hidden nodes, and graphics of difference ( 5 times) between them

Later the other two methods, which are fractal and wavelet based using domain clusters in each case, have compared and evaluated the results with ANN method for the normal ECG signal, it can be seen in Figure 3 and 4. In most cases, the three methods which are neural network, fractal and wavelet, gave very similar average PRD results as $2.77 \%, 3.2 \%$, and $3.0 \%$ respectively. 


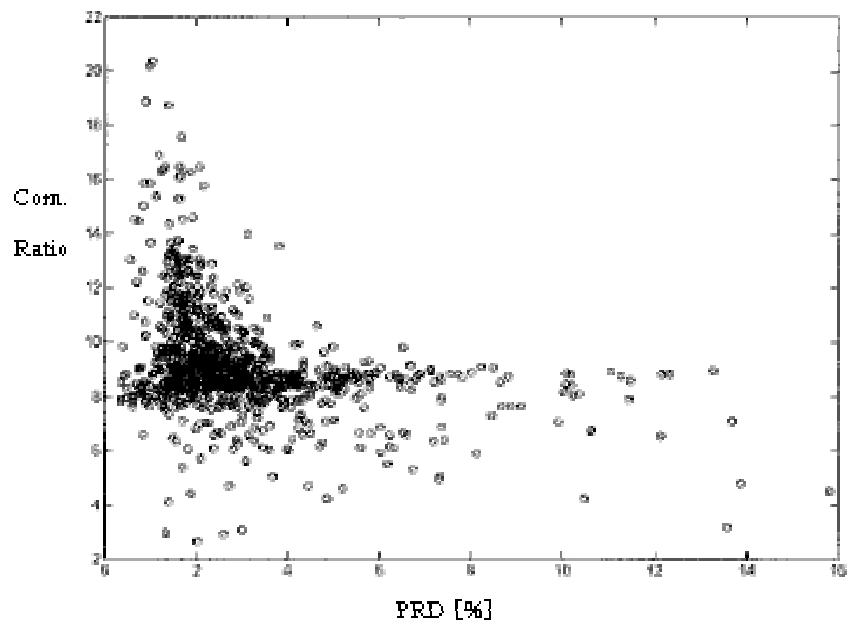

Fig. 3. Resulted values of compression ratio

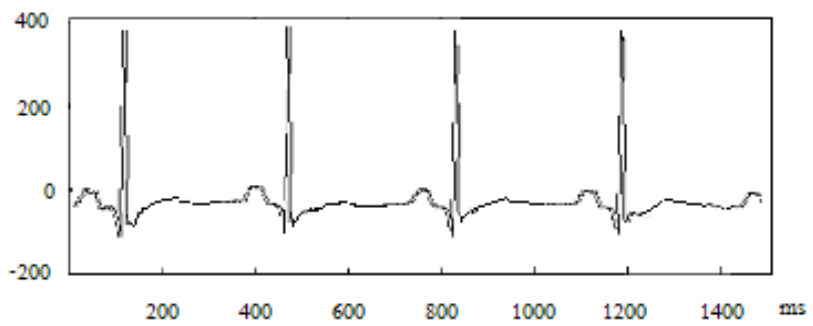

(a)

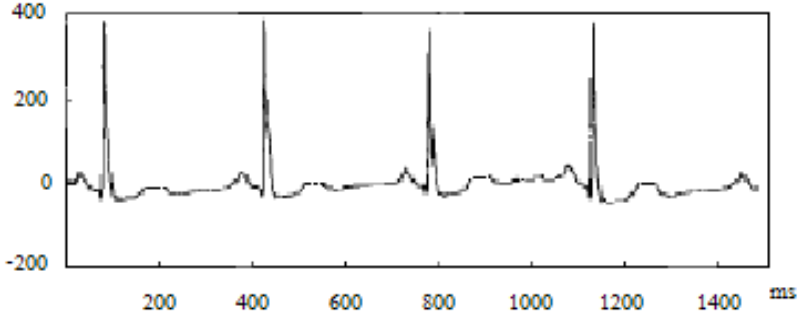

(b)

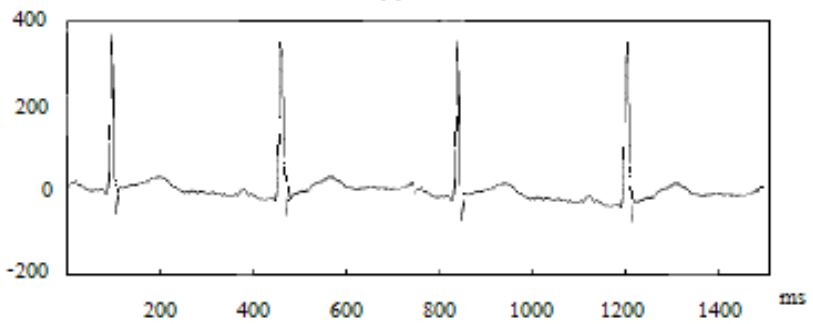

(c)

Fig. 4. Reconstructed ECG signals for (a) neural network (b) fractal and (c) wavelet 


\section{Conclusion}

A hierarchical neural network compression has demonstrated on ECG signals. It is also compared with the two efficient compression methods. Comparing the results with these methods, it can be seen that proposed method is very useful for the either compression ECG and the other biomedical signals. But this neural network method needs long time training for compression. So, this disadvantage should be reduced by using short training time algorithm.

The fractal method has the benefit of fast decoding, but the basic encoder is slow. However, using domain clustering to reduce searching one obtains two decades of complexity reduction without any fatal quality loss. This method should be improved by more optimal parameter representation.

The wavelet-based method can be used and considered as a special case of sub band signal processing. The use of orthonormal base of functions can sufficiently meet the requirement of exact reconstruction. Its quantization step corresponds to the range of coefficients values for fixed word length and implies resulting error.

\section{References}

1. Jalaleddine, S.T.S. et al., ECG Data Compression Techniques - A Unified Approach, IEEE Transactions on Biomedical Eng., Vol. BME-37, pp. 329-343, April (1990)

2. Allen, V.A. and Belina, J., ECG Data Compression Using the Discrete Cosine Transform (DCT), Computers in Cardiology Proceedings, (1992)

3. Thakor, N. et al, A Multi-resolution Wavelet-based ECG Data Compression Algorithm, IEEE Computer Society Press, pp.393-396, (1993)

4. Oien, G.E. et al., "Fast Fractal-Based ECG Signal Compression", CD-ROM Proceedings of the $18^{\text {th }}$ Annual International Conference IEEE Engineering in Medicine and Biology Society, 31 October-3 November 1996, Amsterdam, The Netherlands

5. Provaznik, I. and Kozumplik, J., Wavelet-Based Compression of ECG Signals, CD-ROM Proceedings of the $18^{\text {th }}$ Annual International Conference IEEE Engineering in Medicine and Biology Society, 31 October-3 November 1996, Amsterdam, The Netherlands

6. Karlik, B., Aydin, S., Kilic, I., Comparing Artificial Neural Network (ANN) Image Compression Technique with Different Image Compression Techniques, Mathematical \& Computational Applications, vol. 1, no. 1, pp. 165-171, (1996)

7. Kocyigit, Y., Korurek, M., Karlik, B., ECG Data Compression by Artificial Neural Networks, Proceeding of the ELECO'99, pp.338-340, December, 1-5, 1999, Bursa, Turkey

8. Fisher, Y., Fractal Compression: Theory and Application, Springer-Verlag, (1995)

9. Bertrand, O. et al., Time-Frequency Digital Filtering Based on an Invertible Wavelet Transform: An Application to Evoked Potentials, IEEE Transactions on BME, Vol.41, No.1, pp.77-88, (1994) 